\title{
GESTÃO DA INFORMAÇÃO NA CONVERGÊNCIA ENTRE AMBIENTES COMPARTILHADOS E COWORKING: O QUE A LITERATURA EVIDENCIA
}

\author{
INFORMATION MANAGEMENT AND THE \\ CONVERGENCE OF SHARED ENVIRONMENTS AND \\ COWORKING: THE EVIDENCE IN THE LITERATURE
}

\author{
Paulo Isnarda \\ Ana Cardosob \\ Eric Ferreira Correioc
}

\begin{abstract}
RESUMO
Introdução: $O$ crescente uso de ambientes compartilhados de trabalho na contemporaneidade tem se tornado objeto de análise no meio acadêmico, com destaque para o espaço de coworking, por meio de pesquisas que buscam averiguar as causas e as consequências destas práticas. Objetivo: Identificar pela literatura pesquisas sobre as relações entre coworking e Ambiente Compartilhado dada a importância que cada temática tem separadamente no cenário de constantes mudanças organizacionais e comportamentais, destacando em seguida aspectos da gestão de conhecimento. Metodologia: Essa identificação foi realizada por meio de uma análise quantitativa das publicações contidas na base de dados SCOPUS incluída no portal de periódicos da CAPES. A verificação foi feita por meio dos descritores presentes no texto dos artigos, acrescida de uma análise temporal e da identificação dos principais autores e periódicos que se dedicaram aos temas para alcançar um índice de impacto relevante. Resultados: Foi encontrado o número considerável de 12.020 pesquisas científicas que apresentaram de maneira separada os temas Coworking e Ambiente Compartilhado. Conclusões: O resultado do estudo aponta a existência de uma lacuna na integração dos temas, e permite uma harmonização de conceitos.
\end{abstract}

Descritores: Compartilhamento de Conhecimento. Ambiente Compartilhado. Coworking. Gestão da Informação.

a Doutorando no Programa de Pós-Graduação em Sistemas de Informação e Gestão do Conhecimento da Universidade FUMEC. Docente de Graduação e Lato Sensu na PUC Minas, PREPES, IEC, PUC Master e FEAMIG. Email: ppisnard@yahoo.com.br

b Docente do Programa de Pós-Graduação em Sistemas de Informação e Gestão do Conhecimento da Universidade FUMEC. Email: ana.cardoso@fumec.br

c Doutor em Sistemas de Informação e Gestão do Conhecimento pela Universidade FUMEC. Email: eric.p.f@gmail.com 


\section{INTRODUÇÃO}

O crescente recurso ao coworking e a ambientes compartilhados de trabalho na contemporaneidade tem se tornado objeto de análise entre acadêmicos, por meio de pesquisas que buscam averiguar as causas e as consequências destas práticas. A proposta do artigo é realizar uma abordagem integrativa derivada da importância de investigação do relacionamento entre coworking e ambiente compartilhado, em função de seu tratamento dissimilar segundo a perspectiva de diferentes autores.

É possível observar que ao longo das últimas décadas a globalização e a mudança tecnológica alteraram as relações entre localização geográfica e as atividades econômicas. Podemos destacar as comunicações que permitem uma reconfiguração das interações entre indivíduos e instituições nos domínios do trabalho (CASTELLS, 2007; WATERS-LYNCH et al., 2016).

Devido ao avanço das tecnologias de informação e comunicação e as novas dinâmicas do mercado de trabalho, o número de pessoas que trabalham remotamente está crescendo exponencialmente. Porém, a liberdade de se trabalhar em casa tem um lado negativo, pois, pode levar ao isolamento profissional, dificultando a colaboração. Para solucionar esse problema, tem aumentado a prática dos coworkings, ou seja, locais em que recursos, ideias e serviços são compartilhados entre seus integrantes. Esses ambientes são considerados elementos sociais complexos, pois, podem ser definidos como escritórios físicos onde os trabalhadores de diversos segmentos independentes partilham como local de trabalho (LEFORESTIER, 2009; CAPDEVILA, 2013).

$O$ objetivo da pesquisa aqui relatada foi verificar a convergência na literatura dos termos coworking e ambientes compartilhados através de uma análise quantitativa das publicações que os correlacionam, destacando em seguida aspectos da gestão de conhecimento.

O artigo tem a seguinte estrutura, esta Introdução (1); seguida pela revisão teórica contemplando conceitos de Coworking e Ambiente Compartilhado (2); pela descrição dos procedimentos metodológicos (3); pela apresentação dos resultados através da análise temporal e as principais 
características das publicações (4); e, finalmente o encerramento pelas Conclusões (5).

\section{REVISÃO TEÓRICA}

Esta seção apresenta os conceitos de Coworking e Ambiente Compartilhado que deram a sustentação teórica para o estudo.

\subsection{COWORKING}

Os estudos sobre coworking tiveram seu início no ano de 2005, quando o termo foi relacionado com as práticas de trabalho compartilhado, mas alguns estudiosos já utilizavam este termo para descrever o trabalho produtivo conjunto, aproveitando o entusiasmo da participação em uma comunidade criativa (POZZEBON, 2011; SPINUZZI, 2012; CAPDEVILA, 2013; UDA; ABE, 2015).

A perspectiva histórica remete a três origens distintas comumente tratadas como práticas de coworking, que se constituem como uma resposta lógica às mudanças nas condições socioeconômicas de trabalho (DANTAS, 2006; GERDENITSCH et al., 2016; LECLERCQ-VANDELANNOITTE; ISSAC, 2016). Segundo Gandini (2015), a primeira delas ocorreu em 2005, na cidade de San Francisco (Califórnia), onde o programador de computador Brad Neuberg citou um novo tipo de espaço de trabalho e o chamou de co-working (com hífen), referindo-se ao trabalho em home office e afirmando que em casa ganha-se maior independência, mas, sofre-se com a solidão e os maus hábitos de não estar em uma comunidade de trabalho. Esta primeira tentativa mostrou-se limitada, sem muitas adesões, mas gerou interesse suficiente para inspirar outros modelos. Assim, outros espaços com estas características foram abertos, sendo chamados de Hat Factory e Citizen Space proporcionando o desenvolvimento dos valores de coworking dentro do contexto em que o trabalho compartilhado ou cooperativo se tornou o foco (BRODIE, 2017).

O termo coworking representa um formato de trabalho com base em cooperação e colaboração alicerçado em tecnologias e não diz respeito apenas ao movimento de se criar espaços físicos de trabalho. Embora o termo tenha 
sido empregado historicamente para descrever atividades de trabalho que incentivam a prática colaborativa entre indivíduos, deriva a ideia de rompimento com o modelo convencional das organizações empresariais e de trabalho contemporâneas, uma vez que coworking representa essencialmente uma nova atmosfera de trabalho conjunto (DANTAS, 2003; POZZEBON, 2011; MORISET, 2014; WATERS-LYNCH et al., 2016).

A conceituação de coworking gera diferentes interpretações entre pesquisadores. A polissemia de definições e inclusive de sintaxe, direciona a busca de convergência para uma conceituação padrão a ser adotada em pesquisas futuras. Exemplos como Coworking, Coworking-spaces, Coworking e "Co Working" são citados na literatura (SPINUZZI, 2012; GANDINI, 2015; BOUNCKEN; REUSCHL, 2016; KOJO; NENONEN, 2016).

Spinuzzi (2012) trata o ambiente de coworking como espaço compartilhado, indicando que propiciam maior possibilidade de cooperação entre os integrantes, mesmo que eles trabalhem em iniciativas independentes. Já Moriset (2014) cita coworking como espaço criativo, o que se diferencia das demais interpretações de compartilhamento de espaço, proporcionando um novo ambiente e consequentemente um novo modelo de negócio. Kojo e Nenonen (2016) corroboram com essas afirmações ao destacarem que coworking se trata efetivamente de um negócio de sucesso e o apontam como uma tendência global.

Aparentes contradições sociais e paradoxos estruturais evocadas pelo conceito de coworking decorrem da ambivalência em torno de questões como espaço físico (localização geográfica) e cooperação (relações de trabalho), para incremento de produtividade. Esta dicotomia permite definir um espaço criativo e difuso que se diferencia das demais interpretações, promovendo um "inovador individualismo" (MORISET, 2014; WATERS-LYNCH et al., 2016).

A Figura 1 representa uma alternativa de configuração de ambiente de coworking, ou seja, em um espaço sem barreiras os grupos se organizam em segmentos que permitem os fluxos comunicacionais: 
Figura 1 - Modelo de estrutura de ambiente de coworking

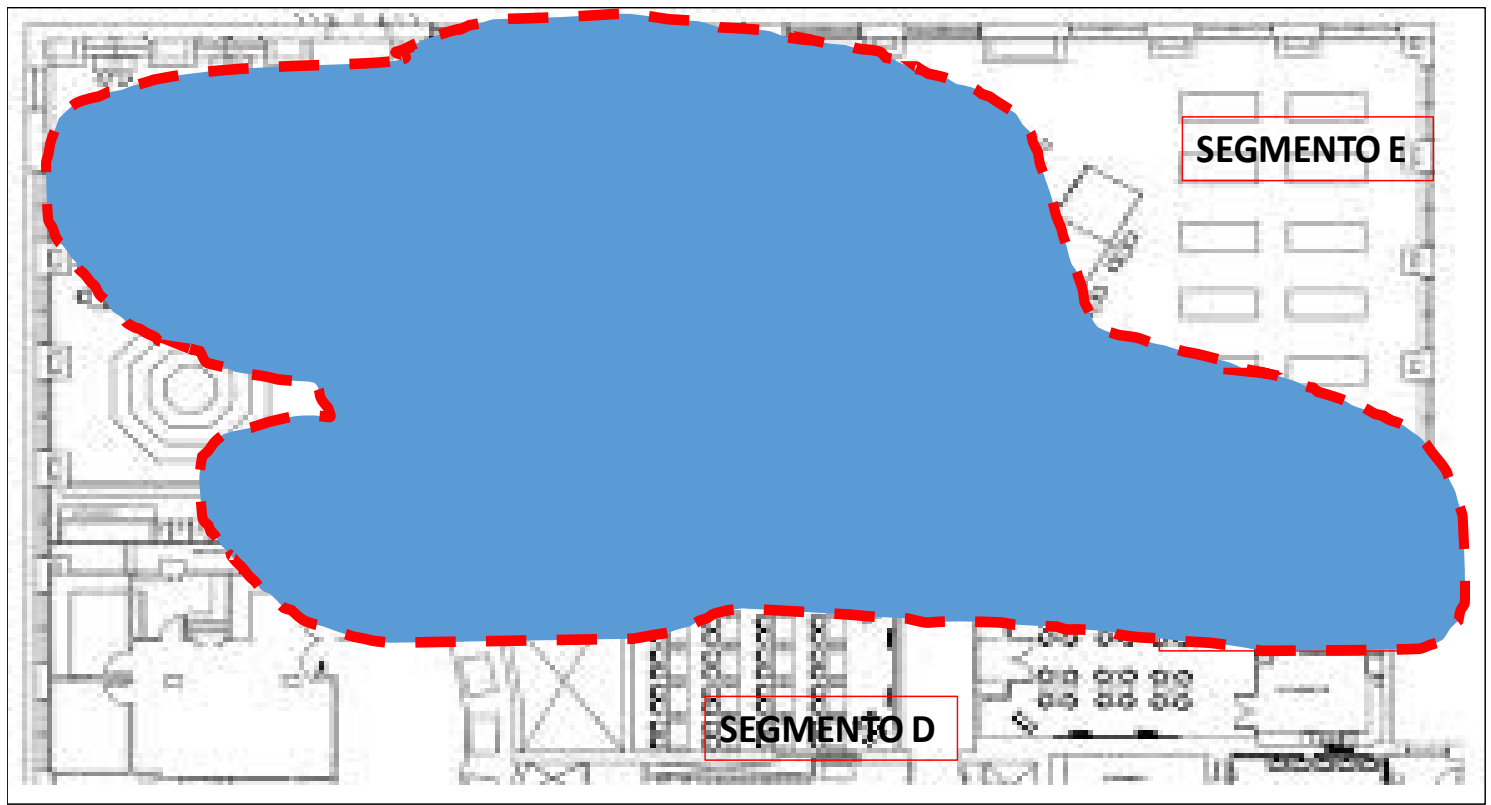

Fonte: Elaborado pelos autores.

A definição de "Lugares Diferentes para Atividades Distintas" (tradução própria para Different Places for Distinct Activities - (SPINUZZI, 2012) é o conceito mais difundido sobre coworking, citando atividades distintas e até contraditórias, sob a ótica de como o coworking concilia interesses dividindo os espaços físicos.

Para Capdevila (2013) esse conceito retrata três configurações diferentes:

Espaços Comunitários - visando atender as comunidades locais, oferecendo espaços com infraestrutura adequada para seus habitantes trabalharem lado a lado;

Unoffices - recria a dinâmica de escritório para trabalhadores independentes com o objetivo de incentivar discussões, reuniões e interações sociais.

Federated Spaces - visam explicitamente promover relações de trabalho e colaboração formal entre os membros.

É possível constatar convergências e discrepâncias entre os conceitos apresentados pelos autores citados. O Quadro 1 sintetiza estas exposições proporcionando o melhor entendimento e a comparação destas definições. 


\section{Quadro 1 - Estabelecimento de comparação}

\begin{tabular}{|c|c|}
\hline AUTOR & SÍNTESE DA DEFINIÇÃO \\
\hline $\begin{array}{c}\text { Granovetter } \\
\text { (1973) }\end{array}$ & $\begin{array}{l}\text { Embora não utilize o termo Coworking, este autor destaca o ambiente social } \\
\text { de trabalho com intensidade de relacionamento } \\
\text { Em ambientes compartilhados de trabalho, a intensidade de um } \\
\text { relacionamento pode estar relacionada com a quantidade de informações que } \\
\text { circulam. O membro organizacional torna-se fundamental no universo } \\
\text { interconectado, pois, compreende aquele indivíduo que se relaciona em redes } \\
\text { de interações e atua exercendo sua agência política na busca de informações. }\end{array}$ \\
\hline $\begin{array}{l}\text { Castells } \\
(2007)\end{array}$ & $\begin{array}{l}\text { Interações entre indivíduos e instituições nos domínios do trabalho } \\
\text { O autor explana sobre a utilização do conceito de interações por meio da } \\
\text { tecnologia entre indivíduos e instituições para a aproximação e captação de } \\
\text { informações, enfatizando o quanto a penetração das tecnologias pode auxiliar } \\
\text { sobre a interações grupais. Essa interação desvela que as organizações } \\
\text { operam em um sistema de rede globalizado onde o fluxo de informações } \\
\text { decorre das relações entre os indivíduos. }\end{array}$ \\
\hline $\begin{array}{c}\text { Pozzebon } \\
(2011)\end{array}$ & $\begin{array}{l}\text { Trabalho produtivo conjunto. A complexidade assumida por esse espaço } \\
\text { compartilhado compreende tanto o ambiente físico quanto o cognitivo. }\end{array}$ \\
\hline $\begin{array}{l}\text { Spinuzzi } \\
\text { (2012) }\end{array}$ & $\begin{array}{l}\text { Espaços compartilhados de trabalho. Enquanto a estratégia de especialização } \\
\text { visa concentrar conhecimentos específicos que tenham sinergia entre si dentro } \\
\text { de um mesmo espaço a fim de melhorar os produtos e serviços dos coworkers, } \\
\text { a estratégia focada em comunidades visa melhor agregar e compartilhar os } \\
\text { conhecimentos, recursos, talentos, a rede de contatos fora do coworking de } \\
\text { cada membro. }\end{array}$ \\
\hline $\begin{array}{l}\text { Groot } \\
(2013)\end{array}$ & $\begin{array}{l}\text { Um efeito paralelo da vida urbana contemporânea, lança um olhar sobre o } \\
\text { coworking considerando-o como um novo formato de trabalho, com enfoque } \\
\text { nos profissionais autônomos que utilizam desse espaço como forma de } \\
\text { ampliação de sua rede de contatos. Espaços de coworking são descritos, } \\
\text { assim, como "lugares de mercado" em que o networking é possível em função } \\
\text { do próprio ambiente físico que estimula o compartilhamento, entendendo-se } \\
\text { que esse ambiente seja uma nova forma de contextualização das situações de } \\
\text { trabalho. }\end{array}$ \\
\hline $\begin{array}{l}\text { Moriset } \\
(2014)\end{array}$ & $\begin{array}{l}\text { Espaços criativos e difusos que se diferenciam das demais interpretações. As } \\
\text { organizações atuais buscam a manutenção de um ambiente promotor da } \\
\text { criatividade, capaz de estimular uma nova visão de mundo, além de favorecer } \\
\text { a instalação de uma cultura voltada à inovação. }\end{array}$ \\
\hline $\begin{array}{l}\text { Gandini } \\
\text { (2015) }\end{array}$ & $\begin{array}{l}\text { Prática de trabalho compartilhado em que há frequentemente cooperação. } \\
\text { Embora o trabalho em ambientes compartilhados ofereça uma comunidade } \\
\text { estável e integrada, os benefícios do compartilhamento de informações estão } \\
\text { fundamentados em uma grande disponibilidade de relacionamentos não } \\
\text { vinculantes e formas de cooperação entre diversos usuários. }\end{array}$ \\
\hline
\end{tabular}

Fonte: Elaborado pelos autores.

Exceto para Spinuzzi (2012) e Moriset (2014), os demais autores não identificam coworking como espaço físico de uso compartilhado, o ambiente pode ser virtual, não necessita coordenadas geográficas comuns. 


\subsection{Ambiente CompaRtilhado}

Novas estruturas organizacionais que exercem influência no desenvolvimento dos negócios a partir da facilitação da comunicação e interação entre os indivíduos foram inseridas nos ambientes de trabalho nos últimos anos (PENN; DESYLLAS; VAUGHAN, 1999). Este tipo de interação funciona como uma forma de identificar um método que facilita o trabalho colaborativo e encontros de negócios coordenados.

Ambientes de trabalho compartilhado oferecem aos usuários a possibilidade de estabelecer networking com profissionais de diversas áreas, fazendo parcerias e participando de cursos de qualificação em um só espaço (DEIJL, 2011; WELCH, 2012). Conforme destacado acima, tais ambientes são elementos sociais complexos, pois, embora os segmentos independentes de trabalhadores compartilhem escritórios físicos, o fluxo de informações que ali circulam permite interpreta-lo como algo mais do que o acesso ao espaço físico e instalações (SPINUZZI, 2012; CAPDEVILA, 2013).

Para Gandini (2015), estes processos influenciam diretamente o ambiente de negócio das empresas, tanto em competitividade como em produtividade. A disseminação das práticas de trabalho compartilhado se traduziu em um negócio de sucesso, proporcionando expectativas elevadas quanto à melhoria das condições de negócios e dos resultados para seus usuários (SPINUZZI, 2012; KOJO; NENONEN, 2016).

Mesmo com ressalvas de alguns críticos focadas particularmente no isolamento e no individualismo, os ambientes compartilhados não são necessariamente apenas uma nova forma de negócio, mas também um efeito paralelo da vida urbana contemporânea (GROOT, 2013). É possível identificar que o ambiente de trabalho compartilhado é uma tendência global, principalmente pelos que o definem como uma prática em que há frequentemente cooperação (intencional ou não) entre os usuários (GRANOVETTER, 1973; BAUMAN; PALLARES-BURKE, 1993; NASCIMENTO; NEVES, 1999; SPINUZZI, 2012; GROOT, 2013; GANDINI, 2015). 


\section{PROCEDIMENTOS METODOLÓGICOS}

Este estudo foi desenvolvido para verificar como essa temática vem sendo difundida nas publicações científicas por meio de um estudo bibliométrico (SALES; GAUDENCIO; NEVES, 2018) com objetivos de caráter exploratório. O levantamento teve início pela busca no Portal CAPES de bases de dados científicas dos termos chaves: coworking e Ambiente Compartilhado.

A base de dados que recuperou maior número de publicações foi a SCOPUS, razão pela qual a revisão da literatura foi feita a partir dos resultados aí obtidos. De posse dos dados da literatura foi então realizada a análise temporal e de descritores para identificar a intensidade de ocorrência dos termos, principais autores, periódicos e áreas de concentração que relacionem o tema.

Com base nos dados identificados, selecionados e analisados foi elaborada uma síntese apresentada a seguir. A análise decorreu dos aspectos privilegiados pelos critérios de busca definidos. Evidentemente outros pontos de vista podem ser desenvolvidos conforme a relevância definida por outros pesquisadores.

\subsection{ANÁlise Individual dAs Temáticas COWORKING E AMBIENTE COMPARTILHADO}

Por meio da busca dos constructos de maneira isolada, percebeu-se que há uma vasta literatura que aborda os temas separadamente, conforme visto nos quadros 2 e 3 . Na pesquisa realizada em setembro de 2018, encontrou-se 12.020 documentos com os termos "coworking" e/ou "ambiente compartilhado".

Quadro 2 - Pesquisa utilizando o termo Coworking separadamente

\begin{tabular}{|l|l|}
\hline Realizada em: & $16 / 09 / 2018$ \\
\hline Termos de pesquisa: & "Coworking" OR "Co-working" \\
\hline Chave de busca: & $\begin{array}{l}\text { TITLE-ABS-KEY ("Coworking" OR "Co-working") AND (LIMIT-TO } \\
\text { (DOCTYPE,"ar")) }\end{array}$ \\
\hline Período: & Superior a 2005 \\
\hline Campos de Pesquisa: & Qualquer \\
\hline Tipo de Publicação: & Só artigos com textos completos \\
\hline Nível da Publicação: & Sem restrição \\
\hline Periódicos: & Bases do Portal de Periódico CAPES \\
\hline Idiomas: & Português e Inglês \\
\hline Retornos: & 9.390 documentos \\
\hline
\end{tabular}

Fonte: Dados da pesquisa. 
Quadro 3 - Pesquisa utilizando o termo ambiente compartilhado separadamente

\begin{tabular}{|l|c|}
\hline Realizada em: & \begin{tabular}{l} 
16/09/2018 \\
\hline $\begin{array}{l}\text { Termos de } \\
\text { pesquisa: }\end{array}$
\end{tabular} \\
\hline Chave de busca: & $\begin{array}{l}3.3 \text { TITLE-ABS-KEY ("Ambiente compartilhado" OR "Shared } \\
\text { environment ") AND (LIMIT-TO (DOCTYPE,"ar")) }\end{array}$ \\
\hline Período: & Superior a 2005 \\
\hline $\begin{array}{l}\text { Campos de } \\
\text { Pesquisa: }\end{array}$ & Qualquer \\
\hline Tipo de Publicação: & Só artigos com textos completos \\
\hline Nível da Publicação: & Sem restrição \\
\hline Periódicos: & Bases do Portal de Periódico CAPES \\
\hline Idiomas: & Português e Inglês \\
\hline Retornos: & 2.630 documentos \\
\hline
\end{tabular}

Fonte: Dados da pesquisa.

Nota-se que estudos sobre coworking são os mais encontrados na base, o que não surpreende pois, esse é um termo mais geral que Ambiente Compartilhado. Pode-se destacar ainda que esse número elevado de publicações ocorre principalmente na área das ciências sociais aplicadas.

\subsection{Análise Relacional das Temáticas Coworking e Ambiente COMPARTILHADO.}

Ao relacionar os termos coworking e ambiente compartilhado, percebeu-se uma diminuição significativa no número de pesquisas retornadas. A relação dos temas coworking e ambiente compartilhado, retornou 59 estudos, conforme o Quadro 4.

Quadro 4 - Pesquisa utilizando os termos Coworking e ambiente compartilhado simultaneamente

\begin{tabular}{|l|l|}
\hline Realizada em: & \begin{tabular}{l}
$17 / 09 / 2018$ \\
\hline Termos de pesquisa:
\end{tabular} \\
\hline Chave de busca: & $\begin{array}{l}3.4 \quad \text { ("Coworking" OR "Co-working") AND ("Ambiente } \\
\text { compartilhado" OR "Shared environment ") }\end{array}$ \\
\hline $\begin{array}{l}3.5 \quad \text { TITLE-ABS-KEY (("Coworking" OR "Co-working") } \\
\text { AND ("Ambiente compartilhado" OR "Shared space") AND } \\
\text { (LIMIT-TO ( DOCTYPE,"ar")) }\end{array}$ \\
\hline Período: & Superior a 2005 \\
\hline Campos de Pesquisa: & Qualquer \\
\hline Tipo de Publicação: & Só artigos com textos completos \\
\hline Nível da Publicação: & Sem restrição \\
\hline Periódicos: & Bases do Portal de Periódico CAPES \\
\hline Idiomas: & Português e Inglês \\
\hline Retornos: & 59 documentos \\
\hline
\end{tabular}

Fonte: Dados da pesquisa. 


\section{ANÁLISE TEMPORAL DOS RESULTADOS E AS PRINCIPAIS CARACTERÍSTICAS DAS PUBLICAÇÕES}

Foi feita uma análise quantitativa dos trabalhos obtidos na revisão da literatura começando pela análise da citação de descritores presentes (ROCHA; GANDRA, 2018) nos campos título, no resumo ou nas palavras-chave dos artigos, objetivando a identificação da maior densidade de palavras-chaves (SILVA; MUDRIK; VIDIGAL, 2018). Após isso, foi realizada uma análise longitudinal com a verificação dos descritores e, posterirormente, a identificação dos indicadores territoriais estatísticos das pesquisas retornadas.

\subsection{DensidAde De TeRmos}

Foi possível desenvolver um mapa empírico da densidade dos termos (RODRIGUES; CERVANTES, 2017), primeiramente, nos resultados isolados com ocorrência dos artigos obtidos na pesquisa. A escala de ocorrência foi colocada como sete vezes. Primeiramente com o termo coworking, conforme a Figura 2:

Figura 2 - Mapa empírico de densidade do termo coworking com ocorrência superior a cinco vezes

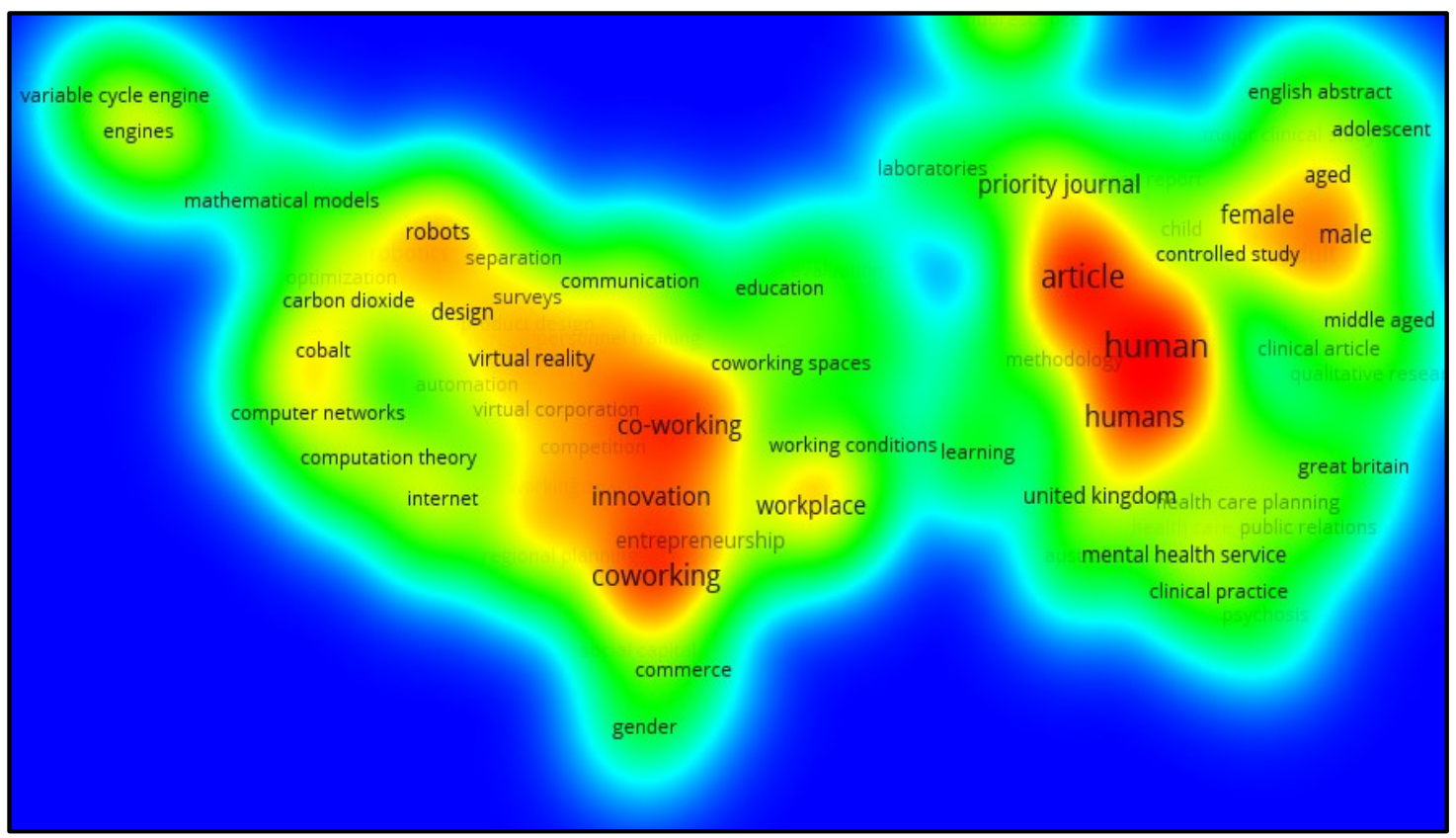

Fonte: Dados da pesquisa. 
Para ocorrência do termo coworking, 9.390 documentos foram contemplados, e posteriormente a análise com o termo Ambiente Compartilhado, na Figura 3.

Figura 3 - Mapa empírico de densidade do termo Ambiente Compartilhado com ocorrência superior a cinco vezes

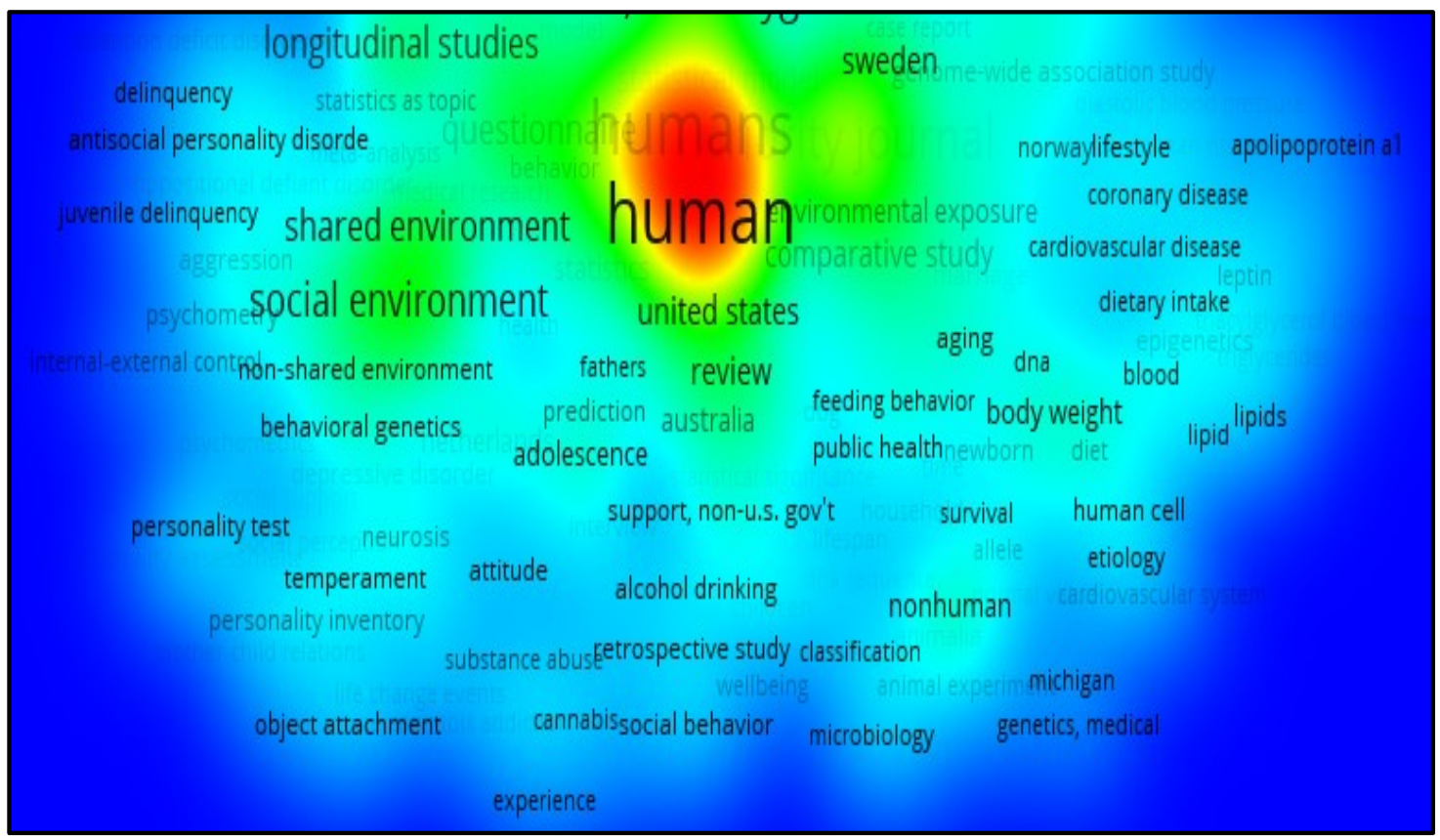

Fonte: Dados da pesquisa.

Neste caso, 2.630 documentos foram contemplados.

Evidencia-se que os termos Coworking e Ambiente Compartilhado e suas respectivas variações, descritas no protocolo de busca, resultam como centrais nas análises de densidade dos termos.

Entretanto, se for feita uma identificação de clusters, surgem novos termos sem relação direta com os termos pesquisados, quando se faz a análise dos temas em conjunto. Aplicou-se um filtro, empregando-se os algoritmos das ferramentas SCIMAT e VOSVIEWER, utilizando-se dos termos que apresentaram maior densidade, os períodos que apresentaram maior incidência de publicações e os países onde houve um maior número de publicações. São estes elementos: Computer Supported Cooperative, Groupware, Coordenation no caso da densidade dos termos; as publicações de 2013 e 2017, para os períodos de publicações; e os países onde foram publicados, conforme a figura 
4 e Gráfico 1, que seguem.

Figura 4 - Link entre os principais termos e Clusters de Termos

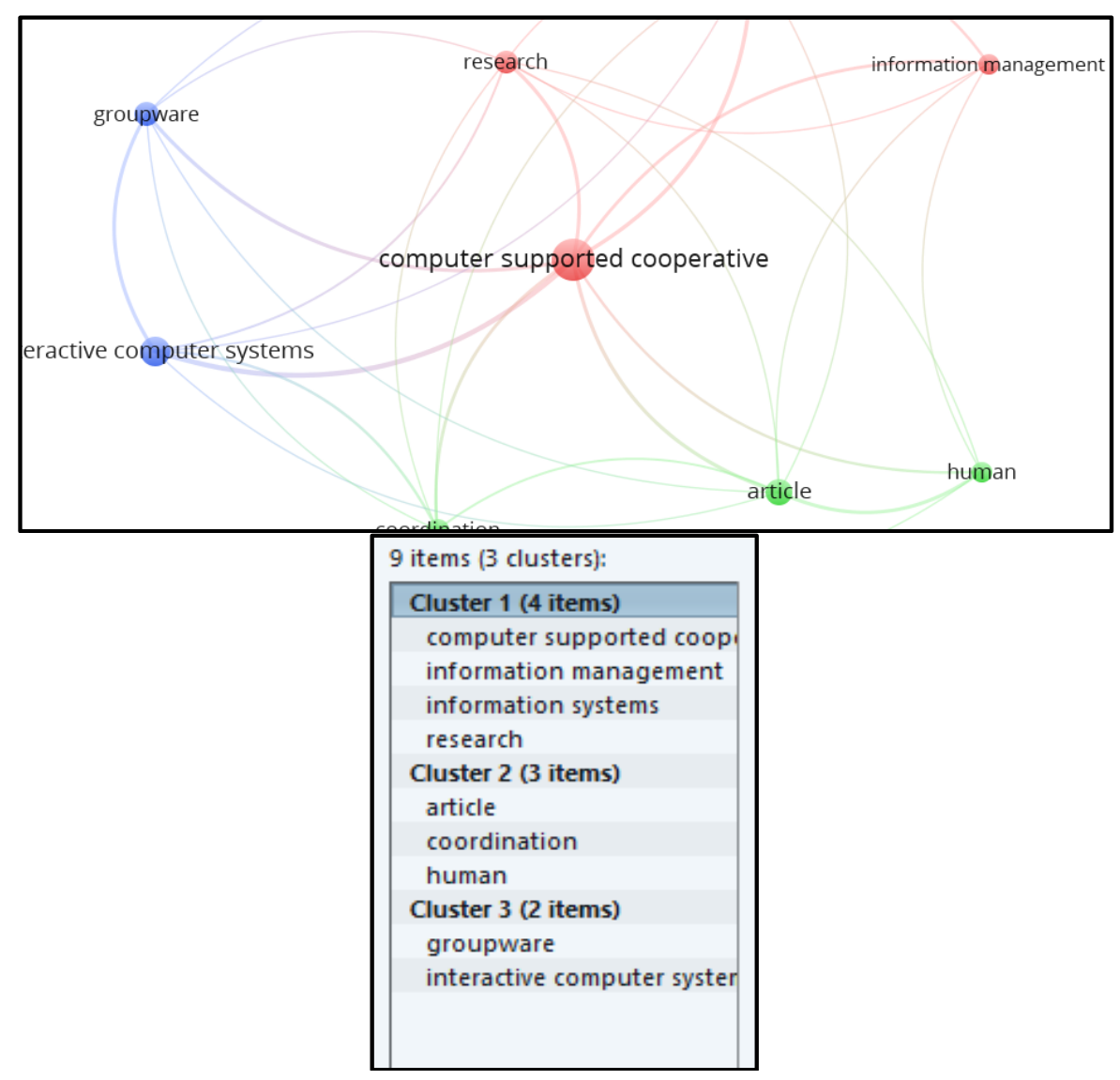

Fonte: Dados da pesquisa.

Vários algoritmos de análise de clusters podem avaliar dados (RODRIGUES; CERVANTES, 2017). No caso deste artigo três representações (diagramas estratégicos, redes de clusters e áreas de evolução) foram utilizadas em conjunto, para permitir uma melhor compreensão dos resultados, através das ferramentas SCIMAT (COBO et al., 2012) e VOSVIEWER (CASTILLOVERGARA; ALVAREZ-MARIN; PLACENCIO-HIDALGO, 2018).

\subsection{AnÁlise Longitudinal com a VerificaÇÃo dos Descritores}

Dentre os documentos obtidos na pesquisa, realizou-se uma análise longitudinal com a verificação da ocorrência dos descritores dentro do intervalo de tempo de 2005 a 2018 a fim de identificar a intensidade das publicações. $O$ resultado dessa análise pode ser visto no Gráfico 1 : 
Gráfico 1 - Verificação dos descritores dentro do intervalo de tempo de 2005 a 2018

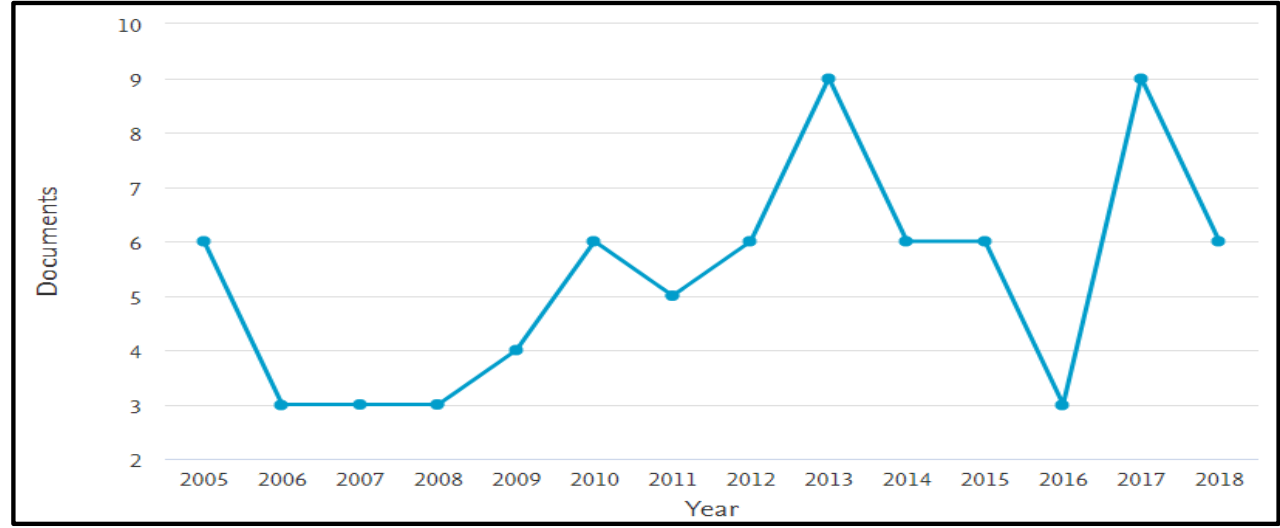

Fonte: Dados da pesquisa.

Identifica-se que os estudos contendo os descritores da pesquisa se intensificaram de 2005 a 2013, ocorrendo um declínio em 2016, entretanto retomando o crescimento em 2017 e 2018.

Assim sendo, as publicações de 2013 e 2017, anos que apresentaram uma maior incidência de publicações também foram utilizados para um detalhamento da pesquisa.

\subsection{INDICADORES TERRITORIAIS ESTATÍSTICOS}

Com o objetivo de identificar os países que mais pesquisam sobre "Coworking" e "Ambiente Compartilhado", utilizaram-se indicadores territoriais estatísticos para classificação das publicações selecionadas, como exposto na Figura 5.

Figura 5 - Países onde incide os maiores números de publicações sobre as temáticas

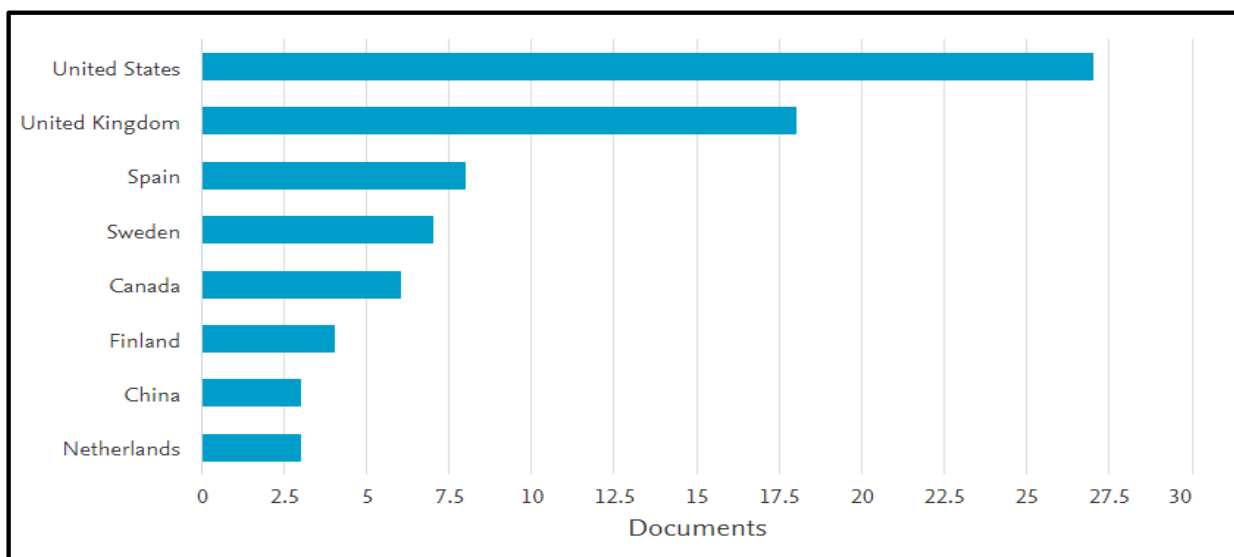

Fonte: Dados da pesquisa. 
Percebe-se que os três países onde houve um maior número de publicações, Estados Unidos, Inglaterra e Espanha, representam mais da metade do total.

\subsection{A GeStÃo dA INFORMAÇÃo NA CONVERGÊnCIA ENTRE AMBIENTES \\ COMPARTILHADOS E COWORKING}

Em um segundo momento do estudo, buscou-se verificar como é tratada a gestão de informação nas 59 publicações que correlacionaram ambientes compartilhados e coworking. Foi feita uma seleção das publicações com base em critérios qualitativos, a fim de permitir uma maior profundidade na análise, o que é demonstrado pela Figura 6:

Figura 6 - Etapas para seleção de artigos

\begin{tabular}{|c|c|}
\hline $9390+2630$ & \begin{tabular}{l} 
COWORKING OU AMBIENTE COMPARTILHADO \\
COWORKING + AMBIENTE COMPARTILHADO \\
TERMOS + ANO + PAÍSES \\
Leitura Completa \\
\hline$?$
\end{tabular} \\
\hline $\begin{array}{c}\text { ANÁLISE QUALITATIVA SOBRE GESTÃO DA INFORMAÇÃO } \\
\text { NA CONVERGÊNCIA ENTRE AMBIENTES } \\
\text { COMPARTILHADOS E COWORKING }\end{array}$ \\
\hline
\end{tabular}

Fonte: Dados da pesquisa.

Após as fases de análise de seleção quantitativa, os artigos foram preparados para uma leitura completa do seu conteúdo. Marconi e Lakatos (2011) citam que uma avaliação mais detalhada se faz necessária para garantir a qualidade dos estudos selecionados. Assim, os nove trabalhos selecionados na fase anterior foram eleitos. vide Figura 7: 
Figura 7 - Relação cronológica de artigos a serem avaliados qualitativamente

\begin{tabular}{|c|c|c|c|}
\hline TÍTULO DO ARTIGO & ANO & AUTOR (ES) & PUBLICADO EM: \\
\hline 1 Knowledge dynamics in localized communities: Coworking spaces as microclusters & 2013 & Capdevila, Ignasi & SSRN Electronic Journal \\
\hline \begin{tabular}{|l} 
Sharing Workspace, Sharing Knowledge : Knowledge Sharing Amongst Entrepreneurs in Jakarta Co-Working \\
2 Spaces
\end{tabular} & 2013 & $\begin{array}{l}\text { Widya, Gita; Soerjoatmodjo, Laksmini; } \\
\text { Bagasworo, Dimas; Joshua, Grimaldie }\end{array}$ & Information Systems Frontiers \\
\hline $\begin{array}{l}\text { Commentary on CoWorking consumers: Co-creation of brand identity, consumer identity, and brand } \\
3 \text { community identity }\end{array}$ & 2017 & Brodie, RoderickJ. & Journal of Business Research \\
\hline 4 Does Board Co-Working Experience Influence Directors' Decisions Toward Internationalization? & 2017 & $\begin{array}{l}\text { Chang, Hsiang-lan Chen Chiao-yi; Hsu, Wen- } \\
\text { tsung }\end{array}$ & $\begin{array}{l}\text { Management International } \\
\text { Review }\end{array}$ \\
\hline \begin{tabular}{|l}
5 The Effectiveness of an Enriched Servicescape Framework on Value-in-use and Behavioural Responses: The \\
Coworking Space Context
\end{tabular} & 2017 & Balakrishnan, Bamini Kpd & $\begin{array}{l}\text { School of Management RMIT } \\
\text { university }\end{array}$ \\
\hline 6 The Hive : Identity Construction within a Coworking Space & 2017 & Abigail, W & $\begin{array}{l}\text { School of Management RMIT } \\
\text { university }\end{array}$ \\
\hline 7 Benefiting from Open Innovation: A Multidimensional Model of Absorptive Capacity & 2017 & Zobel, Ann-kristin & $\begin{array}{l}\text { Journal of Product Innovation } \\
\text { Management }\end{array}$ \\
\hline 8 Working Alone, Together: Coworking as Emergent Collaborative Activity & 2017 & Spinuzzi, C. & $\begin{array}{l}\text { Journal of Business and } \\
\text { Technical Communication }\end{array}$ \\
\hline 9 Why KM projects fail a multi-case analyss & 2017 & Alton, Chua; Alton, Chua & $\begin{array}{l}\text { Journal of Knowledge } \\
\text { Management }\end{array}$ \\
\hline
\end{tabular}

Fonte: Dados da pesquisa.

\subsection{IdentificaÇÃo dA GeStÃo da Informação nOS ARTigos Selecionados}

Com base nestes artigos foi possível identificar e analisar como alguns aspectos qualitativos podem induzir a Gestão da Informação visando gerar conhecimento e a compatibilização de conceitos. Alguns padrões foram identificados, para um entendimento comum dos conceitos que foram agrupados, explicitando a apreciação dos autores:

- Aspectos intangíveis da Gestão da Informação nos ambientes compartilhados (BRODIE, 2017): O autor descreve a necessidade de criação de um ambiente participativo onde o conhecimento tácito, intangível e informal se estabeleça. Entre os aspectos mais relevantes a serem ajustados para um ambiente, segundo o autor (ABIGAIL, 2015; BRODIE, 2017; SPINUZZI, 2012) estão:

Visão: deixar claro para todos, o ramo em que o negócio está implantado e como a empresa espera ser reconhecida pelos stakeholders.

Missão: motivos pelos quais a organização foi concebida e existe,

Crenças: atitudes e valores que darão identidade à organização.

- Processo de identificação de atividades difusoras da Gestão da Informação (SOERJOATMODJO et al., 2015; ZOBEL, 2017): Os autores definem que uma aptidão estratégica para a difusão da informação requer um sistema complexo que em parte compreende qualificações pessoais acrescidas 
de conhecimento incorporado nos sistemas tecnológicos e físicos.

Spinuzzi (2012) complementa a conceituação indicando duas dimensões: os sistemas de gestão, que sustentam o incremento da difusão através de incentivos delineados; e os valores corporativos, que servem para estimular ou inibir a difusão da informação.

- Dimensões de modelos de Gestão de Conhecimento derivados de Gestão da Informação (CHEN; CHANG; HSU, 2017): O modelo oferecido pelos autores parte de uma ótica multidimensional, que evidencia o papel imperioso da área estratégica como garantidora do sucesso na difusão da informação. As dimensões são: cultura organizacional, estrutura organizacional, sistemas de informações, dentre outras.

No modelo apresentado pelo autor verifica-se que as dimensões de sistemas de informação e cultura organizacional apontam para componentes organizacionais tais como: estrutura; pessoas; e ambiente externo, que propiciam uma interface sinérgica entre ambiente compartilhado e coworking.

- Iniciativas de compartilhamento de informação (BALAKRISHNAN, 2017; SOERJOATMODJO et al., 2015): Os autores apresentam elementos que devem ser respeitados para afiançar o sucesso de iniciativas de compartilhamento de informação. Dentre os elementos indicados, destacam-se:

Cultura orientada para o conhecimento: papel mediador da cultura entre a liderança orientada e o sucesso baseado na inovação da empresa.

Infraestrutura tecnológica: aspectos das relações entre inovação tecnológica, infraestrutura tecnológica e crescimento organizacional.

Apoio dos gestores estratégicos: essa prática estratégica e agregadora de valor, densamente arraigada nos mercados e organizações mais amadurecidos, nem sempre se faz presente em organizações de menor porte.

- Motivação (ALTON; WING, 2005): Os autores alegam a importância dos aspectos motivacionais para o envolvimento dos indivíduos e consequente indução no compartilhamento de informações, como plano de carreira e sistema de motivação, podendo direcionar práticas motivacionais a serem desenvolvidas pela gestão da organização em prol do conhecimento.

- Construção de uma organização que faça Gestão da Informação 
(CAPDEVILA, 2013; SPINUZZI, 2012) visando Gestão do Conhecimento (CHEN; CHANG; HSU, 2017; ZOBEL, 2017). A construção de uma organização, sugerida pelos autores, engloba três dimensões interdependentes: infraestrutura organizacional, pessoas e tecnologia. $\mathrm{Na}$ infraestrutura propõem serem implementadas inicialmente variáveis relacionadas ao estilo gerencial. A dimensão pessoas é composta pelas variáveis: modelos mentais, compartilhamento de informações e inovação.

\section{CONCLUSÕES}

Esse estudo teve como objetivo identificar e analisar as possíveis relações entre os constructos coworking e ambiente compartilhado, por meio de uma revisão da literatura filtrada pela Base SCOPUS. De forma complementar, buscou-se identificar a densidade dos termos e os indicadores territoriais estatísticos, além da análise longitudinal com a verificação dos descritores dos estudos obtidos no estudo bibliométrico (ROCHA; GANDRA, 2018).

A pesquisa caracterizou-se como exploratória e de abordagem quantitativa.

Foi possível identificar que, ao se pesquisar os constructos separadamente, um número considerável de publicações científicas é retornado. Ao se analisar as pesquisas relacionando os temas, o número de artigos declina consideravelmente, indicando que a pesquisas que relacionam as temáticas de coworking e ambiente compartilhado ainda estão em fase de consolidação.

A pesquisa contribuiu para esclarecer o entendimento sobre os conceitos de Coworking e Ambiente Compartilhado, principalmente enfatizando a existência de uma baixa integração entre eles, comprovando-se assim a importância do estudo e sua interdisciplinaridade, uma vez que são muitos os conhecimentos necessários à melhor apreensão de ambientes sociais complexos e suas características.

O estudo se limitou a realizar uma análise quantitativa das publicações que abordam as temáticas de forma conjunta e se concentrou na base SCOPUS. Assim, sugere-se para pesquisas futuras avaliar a convergência entre outras 
temáticas aproximadas, bem como verificar a incidência de abordagens qualitativas em estudos sobre sistemas de informação, gestão do conhecimento ou inovação.

\section{REFERÊNCIAS}

ABIGAIL, W. L. The hive: identity construction within a coworking space. 2015. Dissertação (Mestrado em Administração) - School of Management, RMIT University, Melbourne, 2015.

ALTON, C.; WING, L. Why KM projects fail: a multi-case analysis. Journal of Knowledge Management, v. 9, n. 3, p. 6-17, 2005.

BALAKRISHNAN, B. K. The effectiveness of an enriched servicescape framework on value-in-use and behavioural responses: the coworking space context. Tese (Doutorado em Filosofia) - School of Economics, Finance and Marketing, RMIT University, Melbourne, 2017.

BAUMAN, Z.; PALLARES-BURKE, M. L. G. A Sociedade Líquida. Folha de S. Paulo, p. 1-10, 1993.

BOUNCKEN, R. B.; REUSCHL, A. J. Coworking-spaces: how a phenomenon of the sharing economy builds a novel trend for the workplace and for entrepreneurship. Review of Managerial Science, v. 12, n. 1, set. 2016.

BRODIE, R. J. Commentary on coworking consumers: co-creation of brand identity, consumer identity, and brand community identity. Journal of Business Research, v. 70, p. 430-431, jan. 2017.

CAPDEVILA, I. Knowledge dynamics in localized communities: coworking spaces as microclusters. SSRN Electronic Journal, p. 1-18, dez. 2013.

CASTELLS, Manuel. communication, power and counter-power in the network society. International Journal of Communication, v. 1, p. 29, fev. 2007.

CASTILLO-VERGARA, M.; ALVAREZ-MARIN, A.; PLACENCIO-HIDALGO, D. A bibliometric analysis of creativity in the field of business economics. Journal of Business Research, v. 85, p. 1-9, abr. 2018.

CHEN, H. L.; CHANG, H. C. C.; HSU, W. Does board co-working experience influence directors' decisions toward internationalization?. Management International Review, v. 57, n. 1, p. 65-92, fev. 2017.

COBO, M. J.; LÓPEZ-HERRERA, A. G.; HERRERA-VIEDMA, E.; HERRERA, F. SciMAT: a new science mapping analysis software tool, Journal of the 
American Society for Information Science and Technology, v. 63, n. 8, p. 1609-1630, 2012.

DANTAS, M. Informação como trabalho e como valor. Revista Da Sociedade Brasileira de Economia Política, n. 19, p. 44-72, dez. 2006.

DANTAS, M. Informação e trabalho no capitalismo contemporâneo. Lua Nova: Revista de cultura e política, n. 60, p. 05-44, 2003.

DEIJL, C. Two heads are better than one: a case study of the coworking community in the Netherlands. 2011. Dissertação (Mestrado em International Economics) - Erasmus School of Economics, Erasmus University Rotterdam, Rotterdam, 2011.

GANDINI, A. The rise of coworking spaces: a literature review. Ephemera: theory and politics in organizations, v. 15, n. 1, p. 193-205, 2015.

GERDENITSCH, C.; SCHEEL, T. E.; ANDORFER, J.; KORUNKA, C. Coworking spaces: a source of social support for independent professionals. Frontiers in Psychology, v. 7, p. 1-12, abr. 2016.

GRANOVETTER, M. S. The strength of weak ties. The American Journal of Sociology, v. 78, p. 1360-1380, 1973.

GROOT, J. Coworking and networking: how sharing office space contributes to the competitiveness of independent professional. 2013. Dissertação (Mestrado em Estudos Urbanos) - Universiteit van Amsterdam, Amsterdam, 2013.

KOJO, I.; NENONEN, S. Typologies for co-working spaces in Finland - what and how?. Facilities, v. 34, p. 302-313, 2016.

LECLERCQ-VANDELANNOITTE, A.; ISSAC, $H$. The new office: how coworking changes the work concept. Journal of Business Strategy, v. 37, p. 3-9, nov. 2016.

LEFORESTIER, A. The co-working space concept: CINE term project. Gujarat (Índia): Indian Institute of Management Ahmedabad, 2009.

MARCONI, M.; LAKATOS, E. Metodologia do trabalho científico: procedimentos básicos, pesquisa bibliográfica, projeto e relatório, publicações e trabalhos científicos. 7. ed. São Paulo: Atlas: 2011.

MORISET, B. Building new places of the creative economy the rise of coworking spaces. In: GEOGRAPHY OF INNOVATION INTERNATIONAL CONFERENCE, 2., 2014, Utrecht. Anais [...].Utrecht: Utrecht University, 2014. p. 23-25. 
NASCIMENTO, N.; NEVES, J. R. A Gestão do Conhecimento na World Wide Web: reflexões. Perspect. Ciênc. Inf., v. 4, n. 1, p. 29-48, jan./jun. 1999.

PENN, A.; DESYLLAS, J.; VAUGHAN, L. The space of innovation: interaction and communication in the work environment. Environment and Planning B: Planning and Design, v. 26, p. 193-218, 1999.

POZZEBON, M. Relações e práticas que sustentam o trabalho colaborativo e qual o papel das novas tecnologias: GOMA, um espaço de coworking inovador. [S. I.: s. n.], 2011. p. 1-22.

ROCHA, J.; GANDRA, T. Práticas informacionais : elementos constituintes. Inf. \& Inf., v. 23, n. 2, p. 566-595, maio/ago. 2018.

RODRIGUES, M. R.; CERVANTES, B. M. N. Os mapas conceituais e as múltiplas aplicações para a organização e representação do conhecimento. Informatio, v. 22, n. 2, p. 101-121, abr. 2017.

SALES, O.; GAUDENCIO, S.; NEVES, D. Produção científica brasileira em organização e representação da informação: estudo bibliométrico nos periódicos Qualis A. Encontros Bibli: Revista Eletrônica de Biblioteconomia e Ciência da Informação, v. 23, n. 53, p. 16-24, set./dez., 2018.

SILVA, T.; MUDRIK, J.; VIDIGAL, F. A inter-relação entre o processo de inteligência competitiva e a gestão da inovação : proposição de um modelo teórico integrativo. Inf. \& Inf., v. 23, n. 2, p. 427-451, maio/ago. 2018.

SOERJOATMODJO, G. W. L.; BAGASWORO, D. W.; JOSHUA, G.; KALESARAN, T.; VAN DEN BROEK, K. F. Sharing workspace, sharing knowledge: knowledge sharing amongst entrepreneurs in Jakarta co-working spaces. In: INTERNATIONAL CONFERENCE ON INTELLECTUAL CAPITAL AND KNOWLEDGE MANAGEMENT AND ORGANISATIONAL LEARNING, 12., 2015, Bangkok, Thailand. Anais [...].Bangkok, Thailand: Academic Conferences International Limited, 2015. p. 259-267.

SPINUZZI, C. Working alone, together: coworking as emergent collaborative activity. Published in Journal of Business and Technical Communication, v. 26, n. 4, p. 399-441, out. 2012.

UDA, T.; ABE, T. A descriptive statistics on coworking spaces in Japan, Discussion Paper, v. 297, p. 1-40, dez. 2015.

WATERS-LYNCH, J. M.; POTTS, J.; BUTCHER, T.; DODSON, J.; HURLEY, J. Coworking: a transdisciplinary overview. Melbourne: RMIT University, 2016.

WELCH, J. The power of collaboration. Economic Development Journal, v. 11, n. 4, p. 36- 41, 2012. 
ZOBEL, A. Benefiting from open innovation: a multidimensional model of absorptive capacity. Journal of Product Innovation Management, v. 34, n. 3 , p. 269-288, maio 2017.

\title{
INFORMATION MANAGEMENT AND THE CONVERGENCE OF SHARED ENVIRONMENTS AND COWORKING: THE EVIDENCE IN THE LITERATURE
}

\begin{abstract}
Introduction: Studies on shared work environments represent a growing field and it has become an object of analysis in the academic environment, with emphasis on the Coworking space, through researches that seek to investigate the causes and consequences of these practices. Objectives: An initial objective of this paper was to identify in the literature research on the relationship between Coworking and Shared Environment given the importance that each theme has separately in the scenario of constant organizational and behavioral changes, highlighting aspects of knowledge management. Methodology: Quantitative analysis is the method used to find the publications contained in the SCOPUS database included in the CAPES journal portal. The verification was made through the descriptors present in the text of the articles, plus a temporal analysis and the identification of the main authors and periodicals that dedicated themselves to the themes to reach a relevant index of impact. Results: A considerable amount of literature has been published on shared environment. These studies numbered of 12,020 scientific researches were found that presented the Coworking and Shared Environment themes separately. Conclusion: In conclusion this paper indicate certainly these studies show that the existence of a gap in the integration of the themes, and allows a harmonization of concepts.
\end{abstract}

Descriptors: Knowledge Sharing. Shared Environment. Coworking. Information Management

\section{LA GESTIÓN DE LA INFORMACIÓN EN LA CONVERGENCIA ENTRE AMBIENTES COMPARTIDOS Y COWORKING: LO QUE HACE EVIDENTE LA LITERATURA}

\begin{abstract}
RESUMEN
Introducción: Los estudios sobre entornos de trabajo compartidos representan un campo en crecimiento y se ha convertido en un objeto de análisis en el entorno académico, con énfasis en el espacio de coworking, a través de investigaciones que buscan investigar las causas y consecuencias de estas prácticas. Objetivos: Un objetivo inicial de este documento fue identificar en la investigación bibliográfica sobre la relación entre el trabajo compartido y el entorno compartido, dada la importancia que tiene cada tema por separado en el escenario de cambios organizativos y de comportamiento constantes, destacando aspectos de la gestión del conocimiento. Metodología: El análisis cuantitativo es el método utilizado para encontrar las
\end{abstract}


publicaciones contenidas en la base de datos SCOPUS incluida en el portal de la revista CAPES. La verificación se realizó a través de los descriptores presentes en el texto de los artículos, además de un análisis temporal y la identificación de los principales autores y publicaciones periódicas que se dedicaron a los temas para alcanzar un índice de impacto relevante. Resultados: Una cantidad considerable de literatura ha sido publicada en un entorno compartido. Se encontraron estos estudios, con un total de 12,020 investigaciones científicas, que presentaron los temas de Coworking y Ambiente Compartido por separado. Conclusiones: En conclusión, este documento indica ciertamente que estos estudios muestran que existe una brecha en la integración de los temas y permite una armonización de conceptos.

Descriptores: Compartir conocimiento. Ambiente compartido. Coworking. Gestión de la Información. 